\title{
Entwicklung eines PC-gestützten Expertise-Modells für die Pflanzenschutz-Beratung
}

\author{
Angelika Adner, Hermann-Josef Kruse \\ Schering AG, 13342 Berlin, Germany
}

In einem Industriebetrieb stellt sich immer wieder die Aufgabe, für komplizierte und komplexe Sachverhalte, die sich noch dazu immer wieder wandeln, DV-gestützte Modelle zu entwickeln. Der folgende Beitrag handelt davon, wie in der Pflanzenschutz-Sparte der Schering AG mit einem solchen konkreten Problem praktisch umgegangen wurde.

Ein Pflanzenschutz-Berater benötigt für seine Empfehlung zur Pilzbekämpfung an Getreide eine Vielzahl von Informationen, denn eine gezielte Bekämpfungsmaßnahme (sprich: ein effektiver Fungizid-Einsatz) ist von vielen Faktoren abhängig. Es stellt sich nämlich die komplexe Aufgabe, aus einer Vielzahl von Fungiziden mit unterschiedlichen Wirkungen auf verschiedene Pilzkrankheiten ein solches Fungizid (oder eine Kombination) auszuwählen, mit dem ein bestimmter Pilzbefall an einer bestimmten Getreidesorte wirkungsvoll, ökonomisch sinnvoll und ökologisch vertretbar bekämpft werden kann. Hierbei sind viele Nebenbedingungen zu beachten (z.B. Zulassung, Wasserschutzauflage, Einsatztemperatur, erwarteter wirtschaftlicher Schaden).

Daraus ergab sich die Aufgabe, ein PC-Programm zu entwickeln, das den Pflanzenschutz-Beratern bei der Erstellung ihrer Expertisen über Maßnahmen zur Pilzbekämpfung Unterstützung liefern kann. Das Programm sollte anhand feldspezifischer Rahmenbedingungen und Angaben über Art und Stärke des Pilzbefalls eine Bekämpfungsempfehlung unterbreiten. Hierzu war es notwendig, alle für eine Expertise relevanten Informationen zu identifizieren und deren Verknüpfung in einem "Expertise-Modell" abzubilden.

In unserem Vortrag werden wir vor allem darauf eingehen, wie wir an das Projekt herangegangen, auf welche Schwierigkeiten wir gestoßen und wie wir damit umgegangen sind. Die Hauptschwierigkeit bestand im ständigen Ringen um den "optimalen Grad des Pragmatismus". Denn es ging zum einen um die Modellierung eines naturwissenschaftlichtechnischen Sachverhalts, zum anderen mußte aber stets die praktische Beratungssituation im Auge behalten werden.

Die Akzeptanz unserer "Kompromißlösung" beruht u.E. auf folgenden drei Erfolgsfaktoren:

1. Beteiligung aller Know how-Bereiche im Projektteam (Vermeidung von "not invented here"-Effekten).

2. Frühzeitige und ständige Einbindung der Benutzer ("Kunden").

3. Eine schrittweise Programmrealisierung, bei der alle relevanten Unternehmensbereiche konkret an einer Weiterentwicklung des Modells beteiligt sind ("Prototyping"). 